\title{
Impact of Hospitalization on Antihypertensive Pharmacotherapy among Older Persons
}

\author{
Tariq M. Alhawassi ${ }^{1,2} \cdot$ Ines Krass $^{1} \cdot$ Lisa G. Pont ${ }^{3,4}$
}

Published online: 13 August 2015

(c) The Author(s) 2015. This article is published with open access at Springerlink.com

\begin{abstract}
Background Little is known about the impact of hospitalization on antihypertensive pharmacotherapy and blood pressure control in older persons.

Objectives The aim of this study was to explore the impact of hospitalization on the management of hypertension and antihypertensive pharmacotherapy in a cohort of older patients with a documented diagnosis of hypertension.

Methods A retrospective, cross-sectional medical record audit was conducted in a large Australian metropolitan teaching hospital. Patients aged 65 years or older, admitted between January 1st 2010 and December 31st 2010, and with a documented diagnosis of hypertension in their medical record were included in the study. Antihypertensive pharmacotherapy and blood pressure control was compared between admission and discharge. Factors associated with changes to antihypertensive pharmacotherapy were identified.

Results Changes to antihypertensive pharmacotherapy occurred in $39.5 \%(n=135)$ of patients $(n=342)$. On discharge, the proportion of patients receiving antihypertensive pharmacotherapy ( 89.0 vs $85.3 \%, p<0.0001$ ) and the mean number of antihypertensive agents per patient
\end{abstract}

Lisa G. Pont

lisa.pont@mq.edu.au

1 Faculty of Pharmacy, University of Sydney, Sydney, NSW, Australia

2 College of Pharmacy, King Saud University, Riyadh, Saudi Arabia

3 Australian Institute of Health Innovation, Macquarie University, Level 6, 75 Talavera Rd, North Ryde, Sydney, NSW 2013, Australia

4 Sydney Nursing School, University of Sydney, Sydney, NSW, Australia
$(1.7 \pm 1.1$ vs $1.5 \pm 1.1, p<0.0001)$ declined compared with admission. Adverse drug reactions [odds ratio $(\mathrm{OR})=5$, $95 \%$ confidence interval (CI): $2.80-9.34]$ were the main reason documented for antihypertensive pharmacotherapy changes. Patients admitted under the care of medical $(\mathrm{OR}=0.3,95 \%$ CI: $0.17-0.70)$ or surgical $(\mathrm{OR}=0.3$, $95 \%$ CI: 0.12-0.53) specialties were less likely to experience changes to their antihypertensive pharmacotherapy than those treated by gerontology or cardiology teams.

Conclusions Hospitalization has a significant impact on antihypertensive pharmacotherapy. Two out of every five older persons on antihypertensive medications will experience changes to their regimens during admission to hospital with most changes in antihypertensive pharmacotherapy due to adverse drug reactions.

\section{Key points}

Hospitalization has a considerable significant impact on antihypertensive pharmacotherapy among older populations.

Two out of every five older persons using antihypertensive medications experienced changes to their antihypertensive regimens during admission to hospital.

Adverse drug reactions are an important factor associated with changes to antihypertensive pharmacotherapy during hospitalization for antihypertensive medication changes, highlighting the challenges in balancing the benefits and risks associated with the use of these medications in older persons. 


\section{Introduction}

Hypertension is a major risk factor for cardiovascular disease, cerebrovascular disease, and non-cardiovascular complications [1-3]. It is a common chronic condition affecting up to $70 \%$ of the Australian adult population [4, 5]. Hypertension affects three-quarters of the older adult population with a seven-fold higher annual incidence in older compared with younger adults [6]. Pharmacotherapy using a single or a combination of several antihypertensive medications has a pivotal role in the long-term management of hypertension and the prevention of associated morbidity and mortality [7]. Multiple meta-analyses of large well-conducted clinical trials have demonstrated the benefits of pharmacotherapy in the management of hypertension among older and very old populations [8-10]. However, several studies have reported the persistence of suboptimal blood pressure (BP) control among older persons $[11,12]$.

Although older adults represent only $13 \%$ of the Australian population, approximately four out of every ten hospital admissions are for older persons, and persons aged over 65 years account for $50 \%$ of patient bed days [13]. As a common chronic condition, hypertension is routinely managed in the community $[4,5]$. Regular patient review is essential for the management of any chronic condition and can improve patient outcomes. While transitions of care such as hospitalization are often associated with changes in patient care, transitions of care involving changes to medication regimens may represent an opportunity to improve BP control. However, it may also adversely impact BP control associated with changes that may occur during patient hospitalization.

Hospitalization has been reported to have a significant impact on long-term medication use [14]. In the acute care setting, patients with multiple morbidities are managed by multidisciplinary-care teams [15]. Management priorities and treatment goals during hospitalization may be very different to those in primary care. While multidisciplinary care is the ideal for all healthcare, current acute care settings tend to be specialty based, with patients treated by a healthcare team appropriate for their primary presentation. In this context, the control of chronic comorbid conditions such as hypertension may be considered a lower priority. However, little is known about the impact of hospitalization on antihypertensive therapy and BP control among older persons. Therefore, the aim of this study was to explore the impact of hospitalization on hypertension management in older patients. The main objectives of the study were to determine the frequency of changes to antihypertensive medications during hospitalization, the type of changes made, how the changes impact BP control, and to identify risk factors associated with antihypertensive medication changes in a cohort of older patients with a documented diagnosis of hypertension in the acute care setting.

\section{Methods}

\subsection{Study Population}

Patients aged 65 years and over, admitted to a large Australian tertiary metropolitan teaching hospital between January 1st 2010 and December 31st 2010, with a documented diagnosis of hypertension in their medical record were eligible for inclusion in the study. To identify eligible patients, a systematic sampling of every ninth admission throughout the study period was used to ensure that admissions were distributed evenly throughout the year and to overcome any potential seasonal clustering. To allow for adequate medical documentation, only admissions with a duration of at least $48 \mathrm{~h}$ were included in the sample. For patients with multiple admissions during the study period, only the first admission was included in the study. Patients admitted outside the study period, those who did not have a documented diagnosis of hypertension, those for whom the medical records could not be accessed, and those aged less than 65 years were excluded from the study $(n=156)$.

\subsection{Study Design and Covariates}

A retrospective, cross-sectional medical records audit was conducted. Data were extracted from the medical records by a single trained researcher using a pre-defined case record form. All documentation associated with the admission including medical record, drug charts, medical referral letters, hospital admission summary, laboratory reports, pharmacists' note, and medication history and reconciliation form, nursing progress notes, adverse drug reactions (ADRs) summary sheet, and the discharge summary report were reviewed for data collection.

Data collected included demographic information, medical history including documented information on relevant comorbidities, prescribed medications, BP control, and documented diagnosis of either current or a documented history of hypertension on admission. Frailty was determined using the Rockwood scale [16]. Frailty was converted to a dichotomous variable with fit older persons considered as those with a frailty index $($ score $=0)$ and frail patients as those with a (score $\geq 1$ ) [16]. Comorbidity burden was calculated using the Charlson age-adjusted Comorbidity Index [17]. 


\subsection{Antihypertensive Pharmacotherapy}

Antihypertensive medications were defined as those medications recommended for the management of hypertension according to the current National Guideline for the Management of Hypertension [18]. Medicines were coded according to the World Health Organization Anatomic Therapeutic Chemical (ATC) classification system. Antihypertensive medications included angiotensin-converting enzyme inhibitors (ACEI) and angiotensin-II receptor blockers (ARB, ATC C09), calcium channel blockers (CCB, ATC C08), beta blockers (BB, ATC C07) with the exception of sotalol (C07AA07), thiazide and thiazide-like diuretics (ATC C03A, C03B, and C03E), and other antihypertensives (ATC C02), namely methyldopa, moxonidine, prazosin, terazosin, clonidine, and hydralazine. Fixed-dose combination products were considered as two separate medications.

\subsection{Antihypertensive Medication Changes}

Medication use on admission, discharge, and at the time of any actual or potential ADR was recorded. Medication lists on admission were compared with medications on discharge. Differences in antihypertensive medications between the two lists were classified as changes. Changes were then further classified into one of the following mutually exclusive categories: medication commenced, medication ceased, dose increased, dose decreased, or a substitution of a different agent from the same therapeutic class. For each identified change, documented reasons for antihypertensive medications changes were recorded. Reasons for medication changes were considered undocumented if there was no reason recorded for the medication change in the medical record.

\subsection{Hypertension and Blood Pressure Control}

Patients were considered to be at target BP control if they met the current Australia National Guideline for the Management of Hypertension [18]. Comorbidities were taken into consideration for individual patients as specified by the guideline [18]. According to the current Australian guideline, the treatment target for patients with no relevant comorbid conditions is $<140 / 90 \mathrm{mmHg}$ or lower if tolerated, and for those with coronary heart disease, diabetes mellitus, chronic kidney disease, proteinuria, or stroke/transient ischemic attack, the target is $<130 / 80 \mathrm{mmHg}$. [18].

\subsection{Adverse Drug Reactions Causality Assessment}

ADRs were defined according to the World Health Organization definition [19]. Potential ADRs were identified in two ways. ADRs that had been detected and documented by the patients' treating team were identified from the medical records, while a trigger list based on published literature was developed to aid in the identification of potential ADRs that had not been documented in the medical record. The causal relationship between each potential ADR and the suspected medications was assessed using the Naranjo algorithm [20].

\subsection{Study Outcomes}

The main study outcomes were changes to the antihypertensive medication regimen and factors predicting changes in hypertension therapy in older patients with hypertension admitted to an acute care setting.

\subsection{Statistical Analysis}

Data were entered into a custom-built Microsoft Access database for manipulation and analysis was performed using SPSS statistics (Version 20). Continuous variables were presented as means and standard deviation $( \pm \mathrm{SD})$ for normally distributed variables and median and interquartile range (IQR) for those that did not have a normal distribution. Categorical variables were presented as frequencies and percentages. Univariate analyses $(t$ test, Chi-squared test, McNemar's test) were used to test the difference in the sampling distributions of continuous and categorical variables as appropriate. Variables with a $p$ value $<0.1$ and at least 10 patients in case or outcome variables were included in the binary logistic regression model to examine for associations between changes to antihypertensive medication, and this included the following independent variables; length of hospital stay, treating specialty, number of comorbidities, specified comorbidities, number of antihypertensive medications on admission, type of prescribed antihypertensive medication on admission, ADRs. Age and sex were forced into all models as we were interested in examining the impact of patient sex and age and hypertension medication changes. Multicollinearity assumptions were also checked using a tolerance of $>0.1$ and variance inflation factors $<5$. All statistical tests were performed at a significance level of $\alpha=0.05$ and a $95 \%$ confidence interval $(\mathrm{CI})$.

\section{Results}

Medical records of 503 patients were reviewed and of these $347(69.0 \%)$ had a documented diagnosis of hypertension included in their medical record and were included in this study. Patients with a documented diagnosis of hypertension were significantly older than those without and there 
was a higher proportion of female patients (Table 1). The majority of patients were admitted for non-cardiovascular conditions and only three patients were admitted primarily for the management of hypertension

On hospital admission, BP was considered controlled in $128(36.9 \%)$ of the patients with a documented diagnosis of hypertension. Data on BP and BP control prior to the current admission was documented in the medical record for 57 patients $(16.4 \%)$. Of those with prior BP control information, $70.2 \%(n=40)$ were not at target BP prior to hospitalization.

\subsection{Antihypertensive Pharmacotherapy on Admission}

Medication lists on admission represent all medications taken prior to hospitalization. Most patients with a documented diagnosis of hypertension were receiving at least one antihypertensive medication prior to hospitalization. Only $39(11.2 \%)$ were not using any antihypertensive pharmacotherapy on admission.

The most commonly prescribed antihypertensive agents were ARB (38.9\%), ACEI (37.5\%), and CCB (35.4\%) (Table 1). Approximately one third of patients were on monotherapy $(n=131,38 \%)$, with a further $31.0 \%$ $(n=107)$ of hypertension patients on dual therapy and $20.0 \%$ $(n=70)$ on three or more antihypertensive medications. Fiftyseven patients $(18.2 \%)$ were using a fixed-dose antihypertensive combination therapy, and the most common fixed-dose combinations were ARB/hydrochlorothiazide $(n=37$, $11.8 \%)$ and ACEI/hydrochlorothiazide $(n=10,3.2 \%)$.

\subsection{Changes to Antihypertensive Pharmacotherapy}

During hospitalization, 135 (38.9\%) patients with hypertension experienced changes to their antihypertensive medication regimen. On discharge, the proportion of patients with a diagnosis of hypertension who were receiving an antihypertensive agent had declined compared to admission ( 89.0 vs $85.3 \%, p<0.0001)$. In addition, the mean number of antihypertensive agents per patient had also declined $(1.5 \pm 1.1$ vs $1.7 \pm 1.1, p<0.0001)$.

On discharge, ACEI (34.5\%) and CCB (32.3\%) were the most commonly prescribed agents followed by $\mathrm{BB}$ $(31.6 \%)$, ARB (31.6\%), thiazide diuretics $(13.7 \%)$, and other antihypertensive agents $(4.7 \%)$. More patients were discharged on monotherapy (42.4 vs $38 \%, p=0.02$ ), while the proportion of patients on dual antihypertensive medications (28.9 vs $31.0 \%, p=0.36$ ) or three or more antihypertensive medications (15.4 vs $20.0 \%, p=0.003$ ) had declined on discharge. Fewer patients were using fixed-dose antihypertensive combination preparations (10.4 vs $18.2 \%, p<0.0001)$.
The most common change overall was medication cessation. In total, 79 medications were ceased, representing $43.9 \%$ of the total changes. Cessation was also the most common change for each individual drug class. The next most common change was commencement of new antihypertensive medications.

Changes to fixed-dose combination therapy were observed in 21 patients, with the most prevalent change a cessation of fixed-dose therapy and commencement of single-dose therapy $(n=16)$ or cessation of fixed-dose therapy and the patient discharged on no antihypertensive medications $(n=5)$. The most commonly used fixed-dose combinations did not change with $8.1 \%$ of patients using $\mathrm{ARB} /$ hydrochlorothiazide and $2.0 \%$ using ACEI/hydrochlorothiazide on discharge.

On admission, 126 (36.8\%) of all patients with a documented diagnosis of hypertension were at their target BP level. Upon discharge, this figure had increased to $72.0 \%$ $(n=250)$. Of the 126 patients who had controlled BP on admission, 49 patients (38.9\%) experienced changes to their antihypertensive medications. Fifteen patients who were controlled on admission and experienced changes to their medications were not controlled on discharge. Out of 216 patients with uncontrolled BP on admission, 86 (39.8\%) experienced changes to their antihypertensive medications during their admission. Just under half of these $(42.1 \%)$ were then at the target BP level at discharge.

\subsection{Reasons for Antihypertensive Medication Changes}

The main reason for antihypertensive medication changes was suspected ADRs (Table 2). ADRs, where one or more antihypertensive medications were considered causal agents on Naranjo assessment, were significantly associated with changes in antihypertensive medications $(p<0.001)$. In general, the reasons for antihypertensive medication changes were documented in the patients' records, although no documented reason was available for antihypertensive medication changes in 16 patients $(11.9 \%)$.

\subsection{Factors Associated with Changes in Antihypertensive Medications}

On univariate analysis, use of BB or ARB on admission, past or current history of myocardial infarction, admission under cardiology and geriatric treating specialties, occurrence of an ADR during admission, longer length of stay, higher number of antihypertensive medications, impaired renal function (estimated glomerular filtration rate, increased number of comorbidities and comorbid angina, atrial fibrillation, or chronic kidney disease were all 
Table 1 Characteristics of the study population on admission $(n=503)$. Variables are presented as mean $( \pm \mathrm{SD})$, median $( \pm \mathrm{IQR})$, or number (\%)

\begin{tabular}{|c|c|c|c|}
\hline & $\begin{array}{l}\text { Patients with a documented history of } \\
\text { hypertension }(n=347)\end{array}$ & $\begin{array}{l}\text { Patients without a documented history of } \\
\text { hypertension }(n=156)\end{array}$ & $p$ value \\
\hline Mean age (years) & $81.0( \pm 8.4)$ & $78.7( \pm 7.8)$ & 0.004 \\
\hline Number of women & $209(60.2)$ & $86(55.1)$ & 0.284 \\
\hline No. living at home prior to admission & $288(83.0)$ & $127(81.4)$ & 0.703 \\
\hline No. fit (non-frail) & $183(52.7)$ & $86(55.1)$ & 0.630 \\
\hline Mean systolic BP on admission & $143.5( \pm 26.5)$ & $132.9( \pm 21.3)$ & 0.000 \\
\hline Mean diastolic BP on admission & $76.0( \pm 713.7)$ & $73.1( \pm 11.1)$ & 0.014 \\
\hline \multicolumn{4}{|l|}{ No. per treating specialty } \\
\hline Surgical & $99(28.5)$ & $46(29.5)$ & 0.832 \\
\hline Medical & $112(32.4)$ & $59(37.8)$ & 0.263 \\
\hline Cardiology & $83(23.9)$ & $86(16.7)$ & 0.079 \\
\hline Gerontology & $31(8.9)$ & $12(7.7)$ & 0.732 \\
\hline Other & $22(6.3)$ & $13(8.3)$ & 0.450 \\
\hline $\begin{array}{l}\text { Mean estimated glomerular filtration } \\
\text { rate }(\mathrm{mL} / \mathrm{min})^{\mathrm{a}}\end{array}$ & $62.5( \pm 21.7)$ & $71.0( \pm 18.2)$ & 0.000 \\
\hline Median Charlson Comorbidity Index & $2.0( \pm 2.0)$ & $2.0( \pm 3.0)$ & 0.868 \\
\hline \multicolumn{4}{|l|}{ Number with comorbid condition } \\
\hline Angina & $48(13.8)$ & $11(7.1)$ & 0.035 \\
\hline Atrial fibrillation & $84(24.2)$ & $24(15.4)$ & 0.026 \\
\hline Myocardial infarction & $100(28.8)$ & $21(13.5)$ & 0.000 \\
\hline Congestive heart failure & $45(13.0)$ & $15(9.6)$ & 0.303 \\
\hline Depression & $48(13.8)$ & $21(13.5)$ & 1.000 \\
\hline Dementia & $33(9.5)$ & $20(12.8)$ & 0.168 \\
\hline Hyperlipidemia & $141(40.6)$ & $33(21.2)$ & 0.000 \\
\hline Diabetes mellitus & $60(17.3)$ & $13(8.3)$ & 0.009 \\
\hline Current smoker & $21(6.3)$ & $12(7.7)$ & 0.569 \\
\hline Regular alcohol intake & $114(32.9)$ & $55(35.3)$ & 0.611 \\
\hline $\begin{array}{l}\text { Overweight or obese (body mass } \\
\text { index } \geq 25 \mathrm{~kg} / \mathrm{m}^{2} \text { ) }\end{array}$ & $125(36.0)$ & $34(21.8)$ & 0.029 \\
\hline Median number of medications & $8.0( \pm 6.0)$ & $5.0( \pm 5.0)$ & 0.000 \\
\hline $\begin{array}{l}\text { Median number of antihypertensive } \\
\text { medications }\end{array}$ & $2.0( \pm 1.0)$ & $0.0( \pm 0.0)$ & 0.000 \\
\hline \multicolumn{4}{|c|}{ Number using each antihypertensive medication class on admission } \\
\hline ACEI & $130(37.5)$ & $9(5.8)$ & 0.000 \\
\hline ARB & $135(38.9)$ & $3(1.9)$ & 0.000 \\
\hline BB & $107(30.8)$ & $14(9)$ & 0.000 \\
\hline $\mathrm{CCB}$ & $123(35.4)$ & $7(4.5)$ & 0.000 \\
\hline Thiazide and thiazide-like diuretics & $71(20.5)$ & $1(0.6)$ & 0.000 \\
\hline Other antihypertensives & $20(5.8)$ & $1(0.6)$ & 0.007 \\
\hline
\end{tabular}

$B P$ blood pressure, $A C E I$ angiotensin converting enzyme inhibitor, $A R B$ angiotensin receptor blocker, $B B$ beta blocker, $C C B$ calcium channel blocker, $S D$ standard deviation, $I Q R$ interquartile range

a Estimated glomerular filtration rate using the MDRD equation

associated with antihypertensive medication changes (Table 3). Other factors such as smoking, alcohol consumption, living status, number of medications used on admission, use of other antihypertensive classes, patients on fixed-dose combination or patients using two or more antihypertensive medications of the same class, patients with hyper/hypotension episodes during hospital stay, nonmechanical falls, frailty, and patients with comorbid conditions (congestive heart failure, hyperlipidemia, chronic airway disease, asthma, dementia, depression, cancer patients, gout, thyroid disorder) were not associated with medication changes on univariate analysis. 
Table 2 Reasons for changes to antihypertensive medications during hospital admission for patients with a documented diagnosis of hypertension $(n=342)$
Table 3 Factors associated with changes in antihypertensive medications during hospital admission. Factors that remained significantly associated with changes in antihypertensive medication changes on multivariate analysis are highlighted in bold

\begin{tabular}{lc}
\hline Reasons for changes & No. of patients $\left(\%^{\mathrm{a}}\right)$ \\
\hline Adverse drug reaction & $52(38.5)$ \\
Hypotension & $33(24.4)$ \\
Renal impairment or increased risk of renal injury & $23(17.0)$ \\
Poor BP control & $21(15.6)$ \\
Electrolyte disturbance & $11(8.1)$ \\
Cardiovascular comorbidities (Angina/MI/CHF) & $11(8.1)$ \\
Rhythm disturbance & $9(6.7)$ \\
Falls risk & $4(3.0)$ \\
Cerebrovascular accident & $3(2.2)$ \\
Peripheral edema & $3(2.2)$ \\
BP considered controlled & $2(1.5)$ \\
Non-adherence & $1(0.7)$ \\
Palliative care & $1(0.7)$ \\
Gout risk & $1(0.7)$
\end{tabular}

$B P$ blood pressure, $M I$ myocardial infarction, $C H F$ congestive heart failure

${ }^{\text {a }}$ Total does not sum to $100 \%$ because patients may have multiple reasons contributing to each change

\begin{tabular}{|c|c|c|}
\hline Factors & Unadjusted OR $(95 \% \mathrm{CI})$ & Adjusted $\mathrm{OR}^{\mathrm{a}}(95 \% \mathrm{CI})$ \\
\hline Age (years) & $1.0(0.99-1.05)$ & $1.0(0.96-1.03)$ \\
\hline Sex & $0.7(0.43-1.05)$ & $0.7(0.41-1.29)$ \\
\hline Length of stay (days) & $1.0(1.01-1.06)$ & $1.0(0.99-1.05)$ \\
\hline \multicolumn{3}{|l|}{ Treating specialty } \\
\hline Cardiology & $2.7(1.63-4.5)$ & $\mathrm{b}$ \\
\hline Medical & $0.5(0.27-0.84)$ & $0.3(0.17-0.70)$ \\
\hline Surgical & $0.2(0.12-0.44)$ & $0.3(0.12-0.53)$ \\
\hline Geriatric & $0.8(0.34-1.78)$ & $0.7(0.23-1.84)$ \\
\hline Other & $0.1(0.04-0.60)$ & $0.1(0.03-0.60)$ \\
\hline Mean no. of antihypertensive medications & $1.4(1.17-1.77)$ & $1.2(0.91-1.63)$ \\
\hline ARB & $1.6(1.00-2.43)$ & $1.2(0.70-2.21)$ \\
\hline BB & $3.0(1.84-4.77)$ & $2.1(1.08-4.00)$ \\
\hline $\mathrm{ADR}$ & $5.2(3.13-8.44)$ & $5.1(2.80-9.34)$ \\
\hline No. of comorbidities & $1.1(1.00-1.22)$ & $0.9(0.79-1.07)$ \\
\hline eGFR & $0.9(0.97-0.99)$ & $1.0(0.98-1.02)$ \\
\hline Angina & $2.11(1.14-3.93)$ & $1.49(0.68-3.29)$ \\
\hline MI & $2.33(1.44-3.75)$ & $2.09(1.07-3.79)$ \\
\hline $\mathrm{AF}$ & $2.79(1.46-5.36)$ & $1.35(0.72-2.56)$ \\
\hline CKD & $2.08(1.26-3.44)$ & $2.041(0.85-4.91)$ \\
\hline
\end{tabular}

$O R$ odds ratio, $A R B$ angiotensin receptor blocker, $B B$ beta blocker, $A D R$ adverse drug reaction, $e G R F$ estimated glomerular filtration rate, $M I$ myocardial infarction, $A F$ atrial fibrillation, $C K D$ chronic kidney disease, $C I$ confidence interval

a Adjusted for age, sex, length of stay, treating specialty, number of antihypertensive medications, ARB, $\mathrm{BB}, \mathrm{ADR}$, number of comorbidities, renal function, angina, $\mathrm{MI}, \mathrm{AF}$, and $\mathrm{CKD}$

b Cardiology used as the reference
All factors associated with antihypertensive medication changes on univariate analysis where the number of cases was 10 or greater were included in the multivariate model. In the multivariate model, only being treated by cardiology or gerontology specialties, use of a BB or ARB, and a past or current history of myocardial infarction remained significantly associated with antihypertensive medication changes (Table 3). 


\section{Discussion}

This is the first study internationally to demonstrate that changes to antihypertensive medications occur frequently for older patients with comorbid hypertension during hospitalization. Cessation was the most common change to antihypertensive pharmacotherapy observed. ADRs were the primary reason for antihypertensive changes, highlighting the challenges in balancing the benefits and risks associated with the use of these medications in older persons.

Findings from our study show that changes to antihypertensive pharmacotherapy among older inpatients were common, echoing findings of previous studies [21, 22]. Similar to findings by Wang et al. [23] we found that the number of antihypertensive medications decreased following hospitalization. While ACEI were the most commonly prescribed antihypertensive agent on discharge there was a noticeable decline in both ACEI and ARB use following hospitalization. Potential concerns regarding increased risks of side effects such as hypotension, risk of renal injury, and electrolyte disturbances in older persons may be important factors for this. However, both ACEI and ARBs are considered effective agents in the management of hypertension among older adults, particularly in diabetic patients with hypertension, and in general are well tolerated in older patients [24, 25].

In our study, $\mathrm{CCB}$ were the second most common antihypertensive class used at discharge. CCB are often recommended second line after diuretics for the management of hypertension in older individuals, [26, 27] which may be because of perceived high tolerability of CCB in older persons [29] and their role in the management of isolated systolic hypertension [28]. Our findings show a considerably high use of $\mathrm{BB}$, which may be explained by the efficacy of $\mathrm{BB}$ in hypertension management in patients with certain cardiovascular indications such as ischemic heart disease. Commencement was the most common change associated with BB in our study in patients with a past or current history of MI during hospital admission. Increasing use of BB was also reported by a recent US study [30]. In the US study, BB use increased from 12.7 to $43.1 \%(p<0.001)$.

An association between the treating specialty and changes in antihypertensive medications was observed in our study. Despite only three patients admitted primarily for the management of hypertension, patients admitted under the care of cardiology and geriatric teams were more likely to experience changes to their antihypertensive regimens than those managed by medical or surgical teams. De-prescribing by geriatric teams and the management of associated cardiovascular conditions for which antihypertensive medications are indicated may underlie this finding. Other studies have also found that management of very old individuals is influenced by the specialty of the treating healthcare professional [31] with one study reporting that cardiologists were more likely to more comprehensively document ADR and patients medication adherence compared with other specialties [32], both of which were common reasons for medication changes observed in this study.

On admission to hospital, a little over half of the older individuals in this cohort with a current or past documented diagnosis of hypertension were at target BP levels. This percentage increased on discharge to over $70 \%$, highlighting that while the main medication change was cessation of antihypertensive agents, BP control improved during hospitalization. Interestingly, the potential undertreatment of older individuals may also be an issue, with almost $10 \%$ of patients who were not at the target level on admission, having experienced no changes to their medications and still not being at the target level upon discharge. Such under-treatment may warrant further investigation, especially given a study by Maronde et al. that found that underuse of antihypertensive medications following hospital discharge was associated with an increased risk of re-hospitalization [33].

In this study, we found that suspected and actual ADRs were the main reason associated with a change to antihypertensive medications among older patients. Earlier studies have reported that antihypertensive medications are a risk factor for ADRs in general adults, in both the hospital and ambulatory settings [34, 35] and it has been shown that older individuals have a higher risk of experiencing an ADR [36]. However, the size of the risk of antihypertensive-related ADRs in older individuals remains unknown and requires further investigation. ADRs can strongly impact hypertension management and be associated with discontinuation of therapy, [37] leading to subsequent suboptimal BP control [38] and may limit any further required interventions to achieve optimal BP [39]. Thus, further work exploring the risk of antihypertensive ADRs in older populations is needed.

This study has certain limitations, which may affect interpretation of the findings. One limitation was the use of documentation in the medical records to identify persons with hypertension that may have resulted in misclassification of individuals depending on the documentation accuracy. A second limitation was the limited number of patients per variable in the logistic model owing to the exploratory nature of the work. Further work using a large representative sample is needed to confirm the relationships observed in this study. Finally, hospitalization may affect BP control and very limited information on long-term control was available 
for this study. The main strength of this study was the observational design that allowed changes in antihypertensive pharmacotherapy occurring in actual clinical practice to be quantified. Furthermore, this study examined a range of specialties rather than just focusing on patients with hypertension admitted under cardiologists.

\section{Conclusion}

Hospitalization has a significant impact on antihypertensive pharmacotherapy. Two out of every five older persons taking antihypertensive medications will experience changes to their regimens during admission to hospital. While such changes were associated with improved BP, the association between medication changes, ADRs, and other side effects observed in this cohort highlights the need for patient education and better/increased communication with other healthcare professionals regarding medication changes during hospitalization.

\section{Compliance with Ethical Standards}

Funding Mr. Alhawassi received an unrestricted $\mathrm{PhD}$ scholarship from King Saud University.

Conflicts of interest The authors have no conflicts of interest to declare relevant to this study.

Ethical approval This study was approved by the Northern Sydney Central Coast Health Human Research Ethics Committee protocol number (1105-147 M).

Open Access This article is distributed under the terms of the Creative Commons Attribution-NonCommercial 4.0 International License (http://creativecommons.org/licenses/by-nc/4.0/), which permits any noncommercial use, distribution, and reproduction in any medium, provided you give appropriate credit to the original author(s) and the source, provide a link to the Creative Commons license, and indicate if changes were made.

\section{References}

1. Prospective Studies Collaboration. Age-specific relevance of usual blood pressure to vascular mortality: a meta-analysis of individual data for one million adults in 61 prospective studies. Lancet. 2002;360(9349):1903-13.

2. Alter M, Friday G, Lai SM, O'Connell J, Sobel E. Hypertension and risk of stroke recurrence. Stroke. 1994;25(8):1605-10.

3. Tylicki L, Rutkowski B. Hypertensive nephropathy: pathogenesis, diagnosis and treatment. Pol Merkur Lekarski. 2003;14(80): 168-73.

4. Harrison C, Britt H, Miller G, Henderson J. Prevalence of chronic conditions in Australia. PloS One. 2013;8(7):e67494. doi:10. 1371/journal.pone.0067494.

5. Britt H, Harrison C, Miller G, Knox S. Prevalence and patterns of multimorbidity in Australia. Med J Aust. 2008;189(2):72-7.

6. Tanamas SK, Magliano DJ, Lynch B, Sethi P, Willenberg L, Polkinghorne KR, et al. AUSDIAB 2012. The Australian diabetes, obesity and lifestyle study [Internet]. Melbourne: Baker IDI Heart and Diabetes Institute. 2013. Available from: http:// www.bakeridi.edu.au/Assets/Files/Baker\%20IDI\%20Ausdiab\%20 Report_interactive_final.pdf. Accessed 1 Aug 2015.

7. Neal B, MacMahon S, Chapman N. Blood Pressure Lowering Treatment Trialists' Collaboration. Effects of ACE inhibitors, calcium antagonists, and other blood-pressure-lowering drugs: results of prospectively designed overviews of randomised trials. Blood Pressure Lowering Treatment Trialists' Collaboration. Lancet. 2000;356(9246):1955-64.

8. Staessen JA, Gasowski J, Wang JG, Thijs L, Hond ED, Boissel JP, et al. Risks of untreated and treated isolated systolic hypertension in the elderly: meta-analysis of outcome trials. Lancet. 2000;355(9207):865-72.

9. Mulrow C, Lau J, Cornell J, Brand M. Pharmacotherapy for hypertension in the elderly: review. Cochrane Database Syst Rev. 2000;2. doi:10.1002/14651858.CD000028.

10. Schall P, Wehling M. Treatment of arterial hypertension in the very elderly: a meta-analysis of clinical trials. Arzneimittelforschung. 2011;61(4):221-8.

11. Berlowitz DR, Ash AS, Hickey EC, Friedman RH, Glickman M, Kader B, et al. Inadequate management of blood pressure in a hypertensive population. New Engl J Med. 1998;339(27):1957-63.

12. Mulrow CD, Cornell JA, Herrera CR, Kadri A, Farnett L, Aguilar C. Hypertension in the elderly: implications and generalizability of randomized trials. JAMA. 1994;272(24):1932-8.

13. Australian Institute of Health and Welfare 2014. Australian hospital statistics 2012-13. Health services series no. 54. Cat. no. HSE 145; Canberra:AIHW. 2014. Available from: http://www. aihw.gov.au/WorkArea/DownloadAsset.aspx $? \mathrm{id}=60129547000$. Accessed 1 Aug 2015.

14. Himmel W, Tabache M, Kochen M. What happens to long-term medication when general practice patients are referred to hospital? Eur J Clin Pharmacol. 1996;50(4):253-7.

15. Wagner EH. The role of patient care teams in chronic disease management. BMJ. 2000;320(7234):569-72.

16. Rockwood K, Stadnyk K, MacKnight C, McDowell I, Hebert R, Hogan DB. A brief clinical instrument to classify frailty in elderly people. Lancet. 1999;353(9148):205-6.

17. Charlson ME, Pompei P, Ales KL, MacKenzie CR. A new method of classifying prognostic comorbidity in longitudinal studies: development and validation. J Chronic Dis. 1987;40(5):373-83.

18. National Heart Foundation of Australia (National Blood Pressure and Vascular Disease Advisory Committee). Guide to management of hypertension 2008. Updated December 2010. Available from: http://www.heartfoundation.org.au. Accessed 1 Aug 2015.

19. Edwards IR, Aronson JK. Adverse drug reactions: definitions, diagnosis, and management. Lancet. 2000;356(9237):1255-9.

20. Naranjo CA, Busto U, Sellers EM, Sandor P, Ruiz I, Roberts EA, et al. A method for estimating the probability of adverse drug reactions. Clin Pharmacol Ther. 1981;30(2):239-45.

21. Viktil KK, Blix HS, Eek AK, Davies MN, Moger TA, Reikvam A. How are drug regimen changes during hospitalization handled after discharge: a cohort study. BMJ Open. 2012;2(6):e001461. doi:10.1136/bmjopen-2012-001461.

22. Müller-Bühl U, Gerold C, Engeser P, Szecsenyi J. Changes in drug therapy following hospital discharge for patients in a general practice: a German incident study. J Public Health. 2009;17(3):217-23.

23. Wang PS, Avorn J, Brookhart MA, Mogun H, Schneeweiss S, Fischer MA, et al. Effects of noncardiovascular comorbidities on antihypertensive use in elderly hypertensives. Hypertension. 2005;46(2):273-9.

24. Thomas GN1, Chan P, Tomlinson B. The role of angiotensin II type 1 receptor antagonists in elderly patients with hypertension. Drugs Aging. 2006;23(2):131-55. 
25. Wing LM, Reid CM, Ryan P, Beilin LJ, Brown MA, Jennings GL, et al. A comparison of outcomes with angiotensin-converting-enzyme inhibitors and diuretics for hypertension in the elderly. New Engl J Med. 2003;348(7):583-92.

26. National Institute for Health Excellence (NICE). Hypertension: management of hypertension in adults in primary care. Clinical guideline CG127. 2011. Available from: http://www.nice.org.uk/ guidance/cg127. Accessed 1 Aug 2015.

27. Mancia G, Fagard R, Narkiewicz K, Redón J, Zanchetti A, Böhm $\mathrm{M}$, et al. $2013 \mathrm{ESH} / \mathrm{ESC}$ guidelines for the management of arterial hypertension: the Task Force for the Management of Arterial Hypertension of the European Society of Hypertension (ESH) and of the European Society of Cardiology (ESC). Eur Heart J. 2013;34(28):2159-219.

28. Staessen JA, Fagard R, Thijs L, Celis H, Arabidze GG, Birkenhäger WH, et al. Randomised double-blind comparison of placebo and active treatment for older patients with isolated systolic hypertension. Lancet. 1997;350(9080):757-64.

29. Monane M, Bohn RL, Gurwitz JH, Glynn RJ, Levin R, Avorn J. The effects of initial drug choice and comorbidity on antihypertensive therapy compliance? Results from a population-based study in the elderly. Am J Hypertens. 1997;10(7):697-704.

30. Bromfield SG, Bowling CB, Tanner RM, Peralta CA, Odden MC, Oparil S, et al. Trends in hypertension prevalence, awareness, treatment, and control among US adults 80 years and older, 1988-2010. J Clin Hypertens. 2014;16(4):270-6.

31. Hajjar I, Miller K, Hirth V. Age-related bias in the management of hypertension: a national survey of physicians' opinions on hypertension in elderly adults. J Gerontol A Biol Sci Med Sci. 2002;57(8):M487-91.

32. Yusuff KB, Tayo F. Does a physician's specialty influence the recording of medication history in patients' case notes? Br J Clin Pharmacol. 2008;66(2):308-12.

33. Maronde RF, Chan LS, Larsen FJ, Strandberg LR, Laventurier MF, Sullivan SR. Underutilization of antihypertensive drugs and associated hospitalization. Med Care. 1989;12:1159-66.

34. Bates DW, Miller EB, Cullen DJ, Burdick L, Williams L, Laird $\mathrm{N}$, et al. Patient risk factors for adverse drug events in hospitalized patients. Arch Intern Med. 1999;159(21):2553-60.

35. Field TS, Gurwitz JH, Harrold LR, Rothschild J, DeBellis KR, Seger AC, et al. Risk factors for adverse drug events among older adults in the ambulatory setting. $\mathrm{J}$ Am Geriatr Soc. 2004;52(8):1349-54.

36. Rende P, Paletta L, Gallelli G, Raffaele G, Natale V, Brissa N, et al. Retrospective evaluation of adverse drug reactions induced by antihypertensive treatment. J Pharmacol Pharmacother. 2013;4(Suppl 1):S47-50. doi:10.4103/0976-500X.120954.

37. Curb JD, Borhani NO, Blaszkowski TP, Zimbaldi N, Fotiu S, Williams W. Long-term surveillance for adverse effects of antihypertensive drugs. JAMA. 1985;253(22):3263-8.

38. Knight EL, Bohn RL, Wang PS, Glynn RJ, Mogun H, Avorn J. Predictors of uncontrolled hypertension in ambulatory patients. Hypertension. 2001;38(4):809-14.

39. Butt T, Branch R, Beesley L, Martin U. Managing hypertension in the very elderly: effect of adverse drug reactions (ADRs) on achieving targets. J Hum Hypertens. 2010;24(8):514-8. 